\title{
ON STRONG COMMUTATIVITY-PRESERVING MAPS
}

\author{
M. S. SAMMAN \\ Received 4 July 2004 and in revised form 4 December 2004
}

We identify some strong commutativity-preserving maps on semiprime rings. Among other results, we prove the following. (i) A centralizing homomorphism $f$ of a semiprime ring $R$ onto itself is strong commutativity preserving. (ii) A centralizing antihomomorphism $f$ of a 2 -torsion-free semiprime ring $R$ onto itself is strong commutativity preserving.

\section{Introduction and preliminaries}

Let $R$ be a ring with center $Z(R)$. We write the commutator $[x, y]=x y-y x,(x, y \in R)$. The following commutator identities hold: $[x y, z]=x[y, z]+[x, z] y ;[x, y z]=y[x, z]+$ $[x, y] z$ for all $x, y, z \in R$. We recall that $R$ is prime if $a R b=(0)$ implies that $a=0$ or $b=0$; it is semiprime if $a R a=(0)$ implies that $a=0$. A prime ring is clearly a semiprime ring. A mapping $f: R \rightarrow R$ is called centralizing if $[f(x), x] \in Z(R)$ for all $x \in R$; in particular if $[f(x), x]=0$ for all $x \in R$, then it is called commuting. A commuting map is centralizing but the converse is not true, in general. It is easy to see that if $f: R \rightarrow R$ is an additive and commuting map, then $[f(x), y]=[x, f(y)]$ for all $x, y \in R$.

A mapping $f: R \rightarrow R$ is called commutativity preserving if $[f(x), f(y)]=0$ whenever $[x, y]=0$. Commutativity-preserving maps have been extensively studied on operator algebras (see $[7,9,11,12,13]$ and the references therein). Many authors have also worked on commutativity-preserving maps on rings (see $[1,2,6,8]$, where further references are also given).

There has also been considerable interest in strong commutativity-preserving maps. A mapping $f: R \rightarrow R$ is called strong commutativity preserving if $[f(x), f(y)]=[x, y]$ for all $x, y \in R$. A strong commutativity-preserving map is commutativity preserving but the converse does not hold, in general.

We recall that an additive map $f$ from a ring $R$ into itself is called an antihomomorphism if $f(x y)=f(y) f(x)$ for all $x, y \in R$. We will follow Herstein [10] for other undefined notations and terminology used here.

In this paper, we mainly study commutativity-preserving and strong commutativitypreserving properties of homomorphisms and antihomomorphisms of certain rings. We 
show (Proposition 2.1) that an epimorphism of a semiprime ring is strong commutativity preserving if and only if it is centralizing. Furthermore, we prove that if $R$ is a 2torsion-free semiprime ring and $f$ is a centralizing antihomomorphism of $R$ onto itself, then $f$ is in fact strong commutativity preserving (Proposition 2.4). These and some other related results are proved in Section 2.

\section{The results}

Proposition 2.1. Let $R$ be a semiprime ring and $f$ an epimorphism of $R$. Then $f$ is centralizing if and only if it is strong commutativity preserving.

Proof. Assume that $f$ is centralizing. Then, by [3, Lemma 2], $f$ is commuting and hence $[f(x), y]=[x, f(y)]$ for all $x, y \in R$. So,

$$
[f(x y), x]=[x y, f(x)]=x[y, f(x)]+[x, f(x)] y=x[y, f(x)]=x[f(y), x] .
$$

That is,

$$
[f(x y), x]=x[f(y), x] \quad \forall x, y \in R .
$$

Also, $[f(x y), x]=[f(x) f(y), x]=f(x)[f(y), x]+[f(x), x] f(y)=f(x)[f(y), x]$. That is,

$$
[f(x y), x]=f(x)[f(y), x] \quad \forall x, y \in R .
$$

By (2.2) and (2.3), we get $f(x)[f(y), x]=x[f(y), x]$. Since $f$ is onto, therefore we have $f(x)[y, x]=x[y, x]$ for all $x, y \in R$. That is,

$$
(f(x)-x)[y, x]=0 \quad \forall x, y \in R .
$$

Replacing $y$ by $u y$ in (2.4) and using (2.4) again, we get

$$
0=(f(x)-x)[u y, x]=(f(x)-x) u[y, x]+(f(x)-x)[u, x] y=(f(x)-x) u[y, x] .
$$

So,

$$
(f(x)-x) u[y, x]=0 \quad \forall x, y, u \in R .
$$

Replacing $x$ by $x+z$ in (2.4), we get

$$
\begin{aligned}
0 & =(f(x)-x)[y, x]+(f(x)-x)[y, z]+(f(z)-z)[y, x]+(f(z)-z)[y, z] \\
& =(f(x)-x)[y, z]+(f(z)-z)[y, x] .
\end{aligned}
$$

So,

$$
(f(x)-x)[y, z]=-(f(z)-z)[y, x] \quad \forall x, y, z \in R .
$$

Equation (2.8) implies that for all $x, y, z, v \in R$, we have

$$
(f(x)-x)[y, z] v(f(x)-x)[y, z]=-(f(x)-x)[y, z] v(f(z)-z)[y, x] .
$$


Putting $u=[y, z] v(f(z)-z)$ in (2.6) and using (2.9), we get

$$
(f(x)-x)[y, z] v(f(x)-x)[y, z]=0 \quad \forall v \in R .
$$

$R$ being semiprime implies that

$$
(f(x)-x)[y, z]=0 \quad \forall x, y, z \in R .
$$

Replacing $y$ by $w y$ in (2.11), we get

$$
0=(f(x)-x)[w y, z]=(f(x)-x) w[y, z]+(f(x)-x)[w, z] y=(f(x)-x) w[y, z]
$$

Thus,

$$
(f(x)-x) w[y, z]=0 \quad \forall x, y, z, w \in R .
$$

Multiplying (2.13) on the left by $[y, z]$ and on the right by $(f(x)-x)$, we get $[y, z](f(x)-$ $x) w[y, z](f(x)-x)=0$ for all $w \in R$. By the semiprimeness of $R$, we get $[y, z](f(x)-$ $x)=0$ and hence by (2.11), we have $(f(x)-x)[y, z]=[y, z](f(x)-x)=0$ for all $x, y, z \in$ $R$. So, by Herstein [10, Lemma 1.1.8], $(f(x)-x) \in Z(R)$. Therefore, $[f(x)-x, y]=0$ for all $x, y \in R$. That is,

$$
[f(x), y]=[x, y] \quad \forall x, y \in R
$$

Replacing $y$ by $f(y)$ in (2.14), and using (2.14) again, we get $[f(x), f(y)]=[x, f(y)]=$ $[x, y]$ for all $x, y \in R$. This proves that $f$ is strong commutativity preserving.

Conversely, assume that $f$ is strong commutativity preserving. Then,

$$
[f(x), f(y)]-[x, y]=0 \quad \forall x, y \in R \text {. }
$$

Replacing $y$ by $x y$ in (2.15) and using the strong commutativity-preserving property of $f$, we get

$$
\begin{aligned}
0=[f(x), f(x y)]-[x, x y] & =[f(x), f(x) f(y)]-[x, x y] \\
& =f(x)[f(x), f(y)]+[f(x), f(x)] f(y)-x[x, y]-[x, x] y \\
& =f(x)[x, y]-x[x, y]=(f(x)-x)[x, y] .
\end{aligned}
$$

So,

$$
(f(x)-x)[x, y]=0 \quad \forall x, y \in R
$$

Replacing $y$ by $z y$ in (2.17) and using (2.17) again, we get

$$
0=(f(x)-x)[x, z y]=(f(x)-x) z[x, y]+(f(x)-x)[x, z] y=(f(x)-x) z[x, y] .
$$


That is,

$$
(f(x)-x) z[x, y]=0 \quad \forall x, y, z \in R .
$$

Replacing $y$ by $f(x)$ in (2.19), we get

$$
(f(x)-x) z[f(x), x]=0 \quad \forall x \in R .
$$

Replacing $z$ by $x z$ in (2.20), we get

$$
\left(f(x) x-x^{2}\right) z[f(x), x]=0 \quad \forall x \in R .
$$

Multiplying (2.20) on the left by $x$, we get

$$
\left(x f(x)-x^{2}\right) z[f(x), x]=0 \quad \forall x \in R .
$$

Subtracting (2.22) from (2.21), we get $[f(x), x] z[f(x), x]=0$ for all $x, z \in R$. Since $R$ is semiprime, therefore, $[f(x), x]=0$ for all $x \in R$. So, $f$ is commuting and hence centralizing.

Remark 2.2. In Proposition 2.1, the implication that $f$ is strong commutativity preserving implying that it is centralizing also follows from Brešar and Miers [7, Theorem 1]; however, the proof in the case of homomorphisms is simple and we have included it here for the sake of completeness. Furthermore, it may be of independent interest.

Remark 2.3. Let $R$ be a ring and $f: R \rightarrow R$ an antihomomorphism. Then clearly, $f$ is commutativity preserving.

The following proposition shows that under some additional assumptions, an antihomomorphism must be strong commutativity preserving.

Proposition 2.4. Let $R$ be a 2-torsion-free semiprime ring and $f$ a centralizing antihomomorphism of $R$ onto itself. Then $f$ is strong commutativity preserving.

Proof. By [5, Proposition 3.1], $f$ is commuting and hence, $[f(x), y]=[x, f(y)]$ for all $x, y \in R$. So, $[f(x y), x]=[x y, f(x)]=x[y, f(x)]+[x, f(x)] y=x[y, f(x)]$. That is,

$$
[f(x y), x]=x[y, f(x)] \quad \forall x, y \in R .
$$

Also, $[f(x y), x]=[f(y) f(x), x]=f(y)[f(x), x]+[f(y), x] f(x)=[f(y), x] f(x)$. That is,

$$
[f(x y), x]=[f(y), x] f(x) \quad \forall x, y \in R .
$$

From (2.23) and (2.24), we get $[f(y), x] f(x)=x[y, f(x)]$; that is, $[f(y), x] f(x)=x[f(y)$, $x]$ for all $x, y \in R$. Now $f$ being onto implies that $[y, x] f(x)=x[y, x]$. So,

$$
[y, x] f(x)=x[y, x] \quad \forall x, y \in R
$$

Replacing $y$ by $u y$ in (2.25), we get $[u y, x] f(x)=x[u y, x]$. That is,

$$
u[y, x] f(x)+[u, x] y f(x)=x u[y, x]+x[u, x] y \quad \forall x, y \in R .
$$


By (2.25) and (2.26), we get $u x[y, x]+[u, x] y f(x)=x u[y, x]+x[u, x] y$. That is, $u x[y, x]+$ $[u, x] y f(x)=x u[y, x]+[u, x] f(x) y$. This implies that

$$
u x[y, x]-x u[y, x]+[u, x] y f(x)-[u, x] f(x) y=0 .
$$

That is,

$$
[u, x][y, x]+[u, x][y, f(x)]=0 .
$$

Using the fact that $f$ is commuting, we get

$$
0=[u, x][y, x]+[u, x][y, f(x)]=[u, x]([y, x]+[f(y), x])=[u, x][y+f(y), x] .
$$

So,

$$
[u, x][y+f(y), x]=0 \quad \forall x, y, u \in R .
$$

Replacing $u$ by $u z$ in (2.30) and using (2.30) again, we get

$$
\begin{aligned}
0=[u z, x][y+f(y), x] & =[u, x] z[y+f(y), x]+u[z, x][y+f(y), x] \\
& =[u, x] z[y+f(y), x] .
\end{aligned}
$$

That is,

$$
[u, x] z[y+f(y), x]=0 \quad \forall x, y, u, z \in R .
$$

Replacing $u$ by $y+f(y)$ in (2.32), we get $[y+f(y), x] z[y+f(y), x]=0$ for all $x, y, z \in R$. Since $R$ is semiprime, we get

$$
[y+f(y), x]=0 \quad \forall x, y \in R .
$$

Rewriting (2.33), we get $0=[y, x]+[f(y), x]=[y, x]+[y, f(x)]=[y, x]-[f(x), y]$. So,

$$
[f(x), y]=[y, x] \quad \forall x, y \in R
$$

That $f$ is strong commutativity preserving follows from (2.34). Indeed, $[f(x), f(y)]=$ $[f(y), x]=[x, y]$ for all $x, y \in R$.

Remark 2.5. Brešar [4, Proposition 4.1] has proved the following result.

Theorem 2.6. Let $R$ be a 2-torsion-free semiprime ring and let $f: R \rightarrow R$ be a centralizing antihomomorphism. Then,

(a) $S=\{x \in R: f(x)=x\} \subseteq Z(R)$,

(b) if $R$ is prime and $f$ does not map $R$ into $Z(R)$, then $S=Z(R)$.

We note that Theorem 2.6 can also be obtained as an application of Proposition 2.4 if $f$ is onto. Thus our proof (below) can be regarded as an alternate argument for Theorem 2.6 which may also be of independent interest. 
Proof. (a) By (2.33), $f(y)+y \in Z(R)$ for all $y \in R$. Therefore, for $z$ in $S, f(z)+z=2 z \in$ $Z(R)$. So, $[2 z, x]=2[z, x]=0$ for all $x \in R$. As $R$ is 2 -torsion-free, so $[z, x]=0$ for all $x \in R$. Therefore, $z \in Z(R)$ and hence $S \subseteq Z(R)$.

(b) Assume that $R$ is prime and let $z \in Z(R)$. If $z=0$, then $f(0)=0$ implies that $0 \in S$. So, assume that $z \neq 0$. Then $f(z)+z \in Z(R), z \in Z(R)$. So, $f(z) \in Z(R)$. Now replacing $x$ by $z x$ in (2.25), we get $[y, z x] f(z x)=(z x)[y, z x]$. That is,

$$
z[y, x] f(x) f(z)+[y, z] x f(x) f(z)=z x z[y, x]+z x[y, z] x .
$$

As $z \in Z(R)$, by (2.35), we get $z[y, x] f(x) f(z)=z x z[y, x]$. That is,

$$
[y, x] f(x) f(z) z=x[y, x] z^{2} \quad \forall x, y \in R, z \in Z(R) .
$$

By (2.25) and (2.36), we get $[y, x] f(x) f(z) z=[y, x] f(x) z^{2}$. That is,

$$
[y, x] f(x)\left(f(z) z-z^{2}\right)=0 \quad \forall x, y \in R, z \in Z(R) .
$$

Since $R$ is prime, then any nonzero central element is not a zero divisor. Hence, if $f(z) z-$ $z^{2} \neq 0$, then $[y, x] f(x)=0$ for all $x, y \in R$. Then by [10, corollary, page 8], either $f(x)=0$ or $x \in Z(R)$. In any case, $f(x) \in Z(R)$ for all $x \in R$, a contradiction. So, $0=f(z) z-z^{2}=$ $(f(z)-z) z$. As $z \neq 0$, therefore by the above argument, $f(z)-z=0$ and hence $z \in S$. So, $Z(R) \subseteq S$ and by (a), we have $Z(R)=S$.

\section{Acknowledgments}

The author is grateful to King Fahd University of Petroleum and Minerals for supporting this research. The author is also thankful to the referees for their valuable comments which improved the paper.

\section{References}

[1] R. Banning and M. Mathieu, Commutativity preserving mappings on semiprime rings, Comm. Algebra 25 (1997), no. 1, 247-265.

[2] H. E. Bell and M. N. Daif, On commutativity and strong commutativity-preserving maps, Canad. Math. Bull. 37 (1994), no. 4, 443-447.

[3] H. E. Bell and W. S. Martindale III, Centralizing mappings of semiprime rings, Canad. Math. Bull. 30 (1987), no. 1, 92-101.

[4] M. Brešar, Centralizing mappings on von Neumann algebras, Proc. Amer. Math. Soc. 111 (1991), no. 2, 501-510.

[5] _ Centralizing mappings and derivations in prime rings, J. Algebra 156 (1993), no. 2, 385-394.

[6]_ Commuting traces of biadditive mappings, commutativity-preserving mappings and Lie mappings, Trans. Amer. Math. Soc. 335 (1993), no. 2, 525-546.

[7] M. Brešar and C. R. Miers, Commutativity preserving mappings of von Neumann algebras, Canad. J. Math. 45 (1993), no. 4, 695-708.

[8] _ Strong commutativity preserving maps of semiprime rings, Canad. Math. Bull. 37 (1994), no. 4, 457-460.

[9] M. D. Choi, A. A. Jafarian, and H. Radjavi, Linear maps preserving commutativity, Linear Algebra Appl. 87 (1987), 227-241. 
[10] I. N. Herstein, Rings with Involution, The University of Chicago Press, Illinois, 1976.

[11] C. R. Miers, Commutativity preserving maps of factors, Canad. J. Math. 40 (1988), no. 1, 248 256.

[12] M. Omladič, On operators preserving commutativity, J. Funct. Anal. 66 (1986), no. 1, 105-122.

[13] W. Watkins, Linear maps that preserve commuting pairs of matrices, Linear Algebra and Appl. 14 (1976), no. 1, 29-35.

M. S. Samman: Department of Mathematical Sciences, College of Scinces, King Fahd University of Petroleum and Minerals, Dhahran 31261, Saudi Arabia

E-mail address: msamman@kfupm.edu.sa 


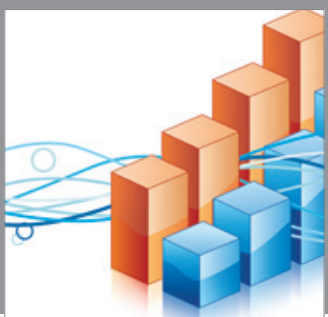

Advances in

Operations Research

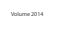

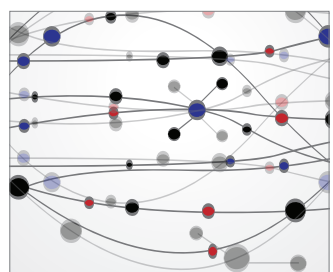

\section{The Scientific} World Journal
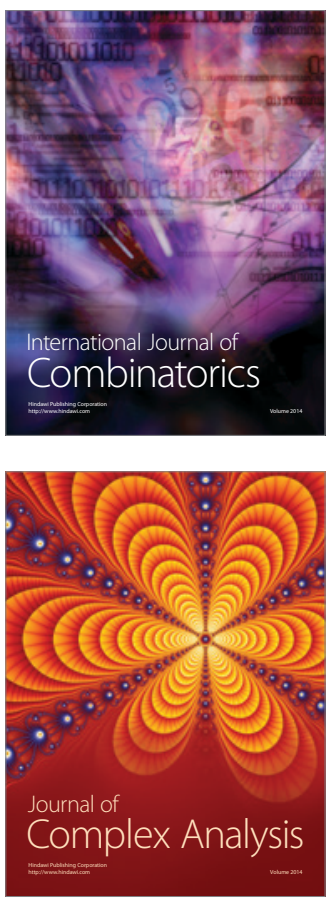

International Journal of

Mathematics and

Mathematical

Sciences
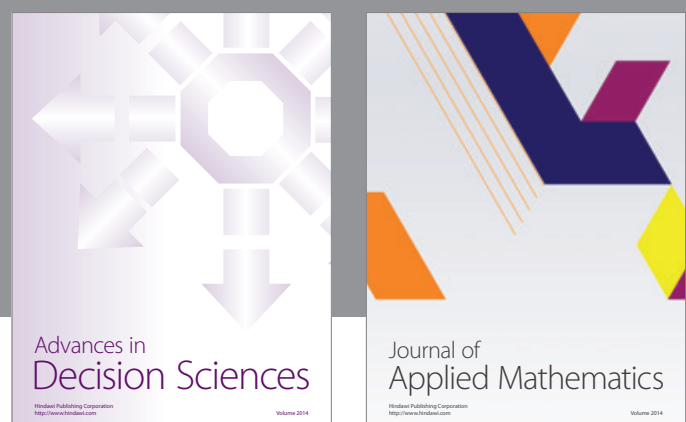

Journal of

Applied Mathematics
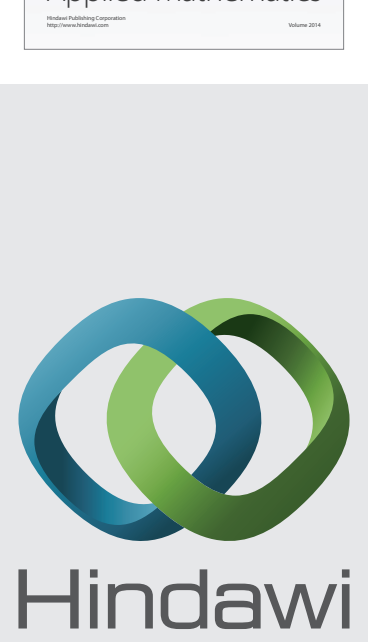

Submit your manuscripts at http://www.hindawi.com
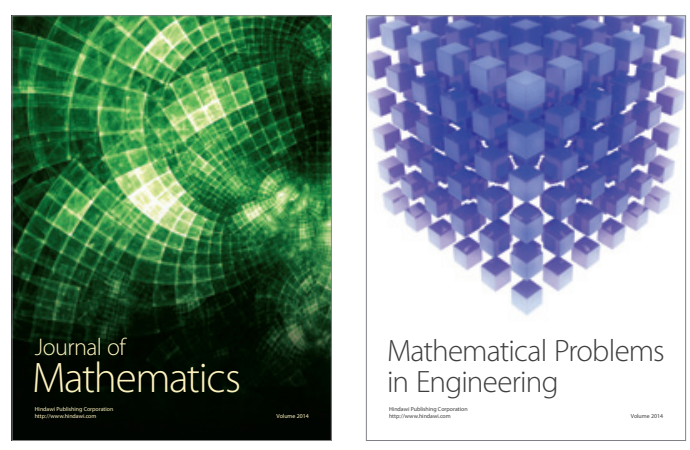

Mathematical Problems in Engineering
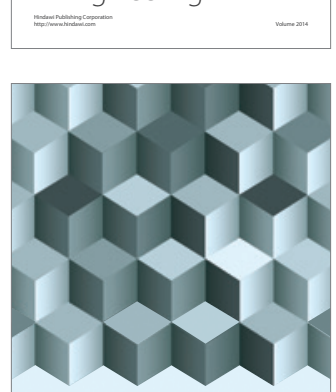

Journal of

Function Spaces
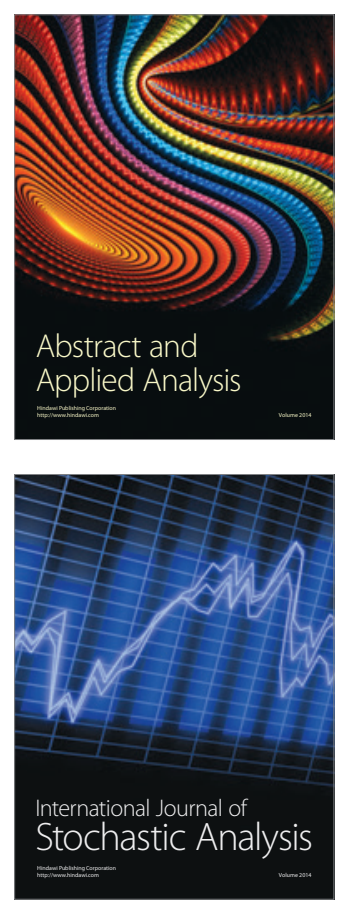

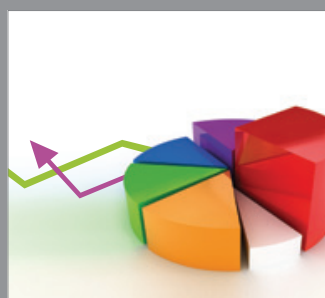

ournal of

Probability and Statistics

Promensencen
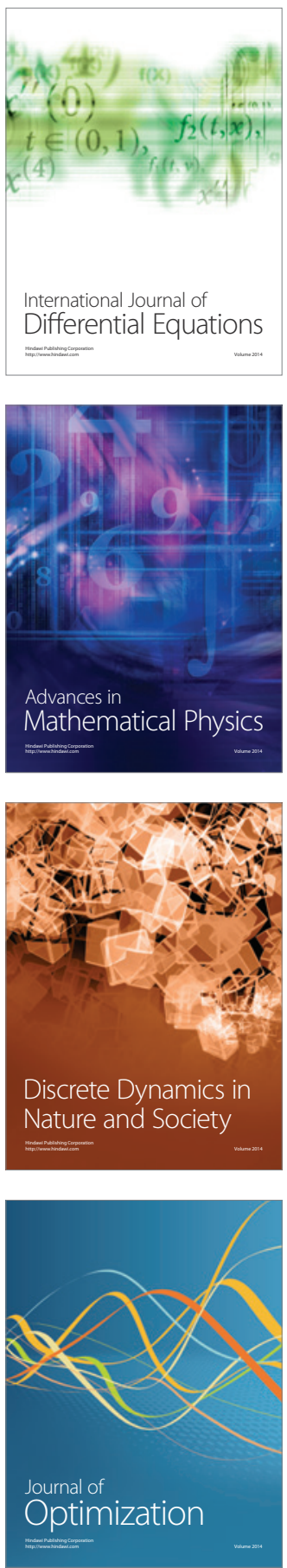